\title{
Realism and the Absence of Rivals
}

\author{
Finnur Dellsén
}

Penultimate draft; please cite published version in Synthese

\begin{abstract}
Among the most serious challenges to scientific realism are arguments for the underdetermination of theory by evidence. This paper defends a version of scientific realism against what is perhaps the most influential recent argument of this sort, viz. Kyle Stanford's New Induction over the History of Science. An essential part of the defense consists in a probabilistic analysis of the slogan "absence of evidence is not evidence of absence". On this basis it is argued that the likelihood of a theory being underdetermined depends crucially on social and historical factors, such as the structure of scientific communities and the time that has passed since the theory first became accepted. This is then shown to serve as the epistemological foundation for a version of scientific realism which avoids Stanford's New Induction in a principled and non-question-begging way.
\end{abstract}

\section{INTRODUCTION}

"Scientific realism" is a label applied to a notoriously large number of views, many of which are in no conflict with one another. The kind of scientific realism that will interest us in this paper is epistemic in that it concerns what we can justifiably believe concerning the posits of scientific theories. More precisely, I shall take epistemic realism to be the view that we are epistemically justified in believing that at least some of the claims made by empirically successful scientific theories - including those that concern 
unobservable entities - are at least approximately true. ${ }^{1}$

Which scientific claims? Well, first of all, realists tend to emphasize empirical success, urging that we should only claim to be justified in believing that empirically successful theories are true. In addition, most realists these days advocate "selective" realisms, in that they urge that only some proper subset of claims made by empirically successful theories are epistemically justified. For example, the entity realism of Cartwright (1983) and Hacking (1983) focuses on the subset of claims about the causally efficacious entities posited by scientific theories. The structural realism of Worrall $(1989,1994)$ focuses on claims that concern the structure of the world in so far as it can be described by purely mathematical models. Finally, the explanationist realism of Kitcher $(1993,2001)$ and Psillos (1999) focuses on the claims that are essential for explaining the theory's empirical success. This paper can be seen as taking the first step towards a different kind of selective realism, one that focuses on claims that enjoy a certain kind of privileged status within scientific communities.

My main objective is to defend this version of epistemic realism against an influential argument that purports to show that scientific theories are underdetermined by the evidence that is meant to support them. As many authors have pointed out - including notably Laudan (1990), Gillies (1993), Kitcher (1993), and Stanford (2013) - there is not a single problem of underdetermination but a cluster of related problems that should not be conflated. One historically significant kind of underdetermination thesis claims that for any scientific theory that posits unobservables, there is an incompatible theory - a "rival" - that is equally well supported given any evidence $E$. In support of this thesis, it has been argued that for any theory $T$, we can construct a rival $T^{*}$ that makes all the same empirical predictions as $T$, and thus no empirical evidence $E$ could possibly distinguish between $T$ and $T^{*}$ (Kukla, 1993). However, as Stanford $(2001,2006)$ points out, this arguably proves too much, in that it would undermine more than just scientific realism. After all, we can construct algorithms of a similar kind for any proposition that purports to be inductively established - be it about unobservable entities posited by scientific theories or the ordinary observ-

\footnotetext{
${ }^{1}$ Since nothing in this paper turns on the distinction between true and approximately true theories, I shall henceforth use "true" to stand for "true or approximately true".
} 
able objects that we can see and touch. So this argument would seem to force us towards a more radical kind of anti-realism not just about unobservable entities, but also about many observable entities - and perhaps even to skepticism about the external world itself. ${ }^{2}$

What would pose a special problem for scientific realism, however, is if it could be argued that successful theories that posit unobservable entities regularly have equally well supported serious rival theories - nonskeptical alternative theories that would be taken seriously by the working scientist. If it could be show that there are rival theories of this sort for most currently accepted scientific theories about unobservables, then epistemic realists could not dismiss underdetermination as "everyone's problem." Accordingly, anti-realists have recently argued that our justification for theories about unobservables is threatened not just by the existence of skeptical alternatives produced by some general algorithm, but rather by serious alternative theories that propose genuinely distinct accounts of the unobservable aspects of reality and that would be taken seriously as such by working scientists. Following Stanford (2001, 2006), let us call this Recurrent, Transient Underdetermination:

RTU: For most successful scientific theories that posit unobservable entities, there exist serious rivals to those theories that are equally well supported given the currently available evidence. ${ }^{3}$

From now on, I shall say that a theory $T$ is underdetermined just in case our current evidence supports some serious rival $T^{*}$ equally well as it supports $T$; and I shall refer to such a rival $T^{*}$ as an underdetermination rival to $T$. Stated in these terms, RTU is the thesis that most successful theories that posit unobservable are underdetermined, i.e. have underdetermination rivals. This is the underdetermination thesis that I shall be concerned with

\footnotetext{
${ }^{2}$ This is not to say that algorithms of this sort would not be a problem for scientific realism as well. The point is rather that the problem would extend also to a host of other philosophical positions, including anti-realist positions such as Stanford's own instrumentalism (which does not deny the possibility of inductive knowledge in general and even allows for the existence of some scientific knowledge concerning unobservable entities and processes (see e.g. Stanford, 2006, 31-33)).

${ }^{3}$ Stanford focuses on scientific claims about "fundamental constituents of the natural world" (Stanford, 2006, 32) rather than those about unobservable objects. In what follows, this difference will not be important.
} 
in the rest of this paper. ${ }^{4}$

How might one argue in favor of RTU? Well, one strategy would be to go through all successful theories that posit unobservable entities and show by example that most of them have underdetermination rivals. However, most of the time we cannot do that, because (as realists like to point out) it is often hard enough to find a single theory that fits the evidence at hand, let alone more than that. (Kitcher, 1993; Psillos, 1999) So, on this strategy, the anti-realist would only undermine the justification for believing a very select group of theories, namely those for which we can find such rivals. While some such rivals have arguably been identified, e.g. by (Glymour, 1977), these examples do not establish the sweeping conclusion that most successful scientific theories have such rivals. Moreover, it's clear that not even the most devout realists would recommend that we believe theories that we know to have underdetermination rivals. (Psillos, 1999) So, if underdetermination is to be a problem for even remotely sophisticated epistemic realisms, then there must be some way to argue that even theories for which we haven't (yet) produced underdetermination rivals are likely to be underdetermined.

That is indeed what anti-realists have tried to do in recent years. Influenced by the classic Pessimistic Meta-Induction (Poincaré, 1952; Putnam, 1978; Laudan, 1981), anti-realists have argued that investigation into the history of science suggests that at least in many cases, scientists have simply failed to conceive of underdetermination rivals to successful scientific

\footnotetext{
${ }^{4}$ In stating RTU I have deliberately been somewhat vague on what counts as a serious rival since the positions and arguments that follow do not turn on precisely how this notion is defined. Two points are worth emphasizing however: (i) For the purposes of this paper, a rival theory is simply a theory that is incompatible with the theory it rivals. Some would argue that a theory doesn't count as a rival theory unless it at least addresses the question answered by the theory it rivals, so that (for example) the negation of a theory doesn't normally count as a rival to the theory, despite being incompatible with it. (Leplin and Laudan, 1993) While I agree (see [reference omitted]), I will operate with the more inclusive definition of "rival theory" in order to ensure that Stanford's argument is not being construed uncharitably. (ii) I am following Stanford (2001, 2006) in characterizing a serious rival to a given theory as a theory that would be viewed by working scientists as a genuine non-skeptical alternative to the theory. This is admittedly somewhat vague since it is not entirely clear what it is for scientists to view a theory as a genuine non-skeptical alternative. Thus one could complain that RTU should be dismissed on the grounds that the alleged problem has not been clearly posed. However, since I do not want to decide the matter on a technicality of this sort, I will operate with Stanford's admittedly somewhat vague notion in what follows.
} 
theories, even when such rivals existed. According to proponents of this argument, this gives us (inductive, empirical) evidence for the conclusion that currently accepted theories, especially the ones that concern unobservable entities, are also likely to have rivals of this sort. In short, we would have reasons to think that most presently accepted scientific theories are underdetermined by the currently available evidence. Although both Hesse (1976) and Sklar (1981) can be read as proposing arguments of this sort, the most prominent recent proponent of the argument is Stanford (2001, 2006, 2009), who calls it the New Induction over the History of Science. ${ }^{5}$

In what follows, I develop a line of response to this argument, undermining the support for RTU. However, let me be upfront about the fact that I think it's clear that there is some truth to RTU, for sometimes we really do have good reasons to suppose that there are underdetermination rivals to our theories - even when no such rivals have been identified. Accordingly, it seems to me that the epistemic realist should look for a way of distinguishing between those theories (and indeed those parts of theories) that are more and less likely to be underdetermined. Using such a distinction, we can argue that the New Induction does not go through for the theories that fall on the less-likely-to-be-underdetermined side of the distinction. So my response to this argument is not to argue it is wholly without merit, but rather to find a suitably restricted set of scientific theories for which it does not apply. I should also emphasize that I will not argue against RTU directly, but rather argue that the New Induction does not succeed in establishing it (for a suitably restricted set of theories).

Many previous authors have suggested structurally similar responses to Stanford's New Induction (or its ancestor, the Pessimistic Meta-Induction), in that they argue that now-discarded theories are relevantly different from currently accepted theories so as not to warrant an induction from the former to the latter. Note that this is not so much $a$ strategy for responding to these arguments as it is the strategy, since any epistemic realist will

\footnotetext{
${ }^{5}$ See also Roush (2005), Magnus (2010), and Wray (2011). Of course, the name and general structure of the argument stems from Laudan's (1981) hugely influential discussion of the Pessimistic Meta-Induction. Nevertheless, for the purposes of this paper it is important not to confuse the two arguments since the argument that follows is concerned specifically with the existence of underdetermination rivals to current scientific theories - an issue on which the Pessimistic Meta-Induction is silent.
} 
have to provide some way of blocking the anti-realist induction from past to present theories. The hard and interesting part of any realist response is to provide a principled and non-question-begging way of drawing the distinction between "good" and "bad" theories such that some significant subset of currently accepted theories fall in the former category whereas now-discarded theories fall in the latter. This paper aims to do precisely that by showing how a relevant distinction can be drawn based on a widely accepted probabilistic approach to epistemology and some uncontroversial facts about how science has evolved over the years. Thus the paper (if successful) provides a sound epistemological foundation for a defensible kind of scientific realism and a new response to the New Induction that is neither ad hoc nor question-begging.

Before we go any further, let me foreshadow the line of argumentation in what follows. The key point for which I will be arguing is that there are certain readily identifiable indicators of when a theory is likely to have unconsidered underdetermination rivals, which may be then used to distinguish between theories that are more and less likely to be underdetermined. In section 3, I discuss these indicators, which concern various sociological and historical factors, such as the length of time a theory has been accepted and the nature of the scientific community that operates with the theory. Using these indicators, I argue (in section 4) that the inductive base of the New Induction is dissimilar to the inductive target in precisely the respect about which the argument makes its conclusion. In order to flesh out this response with as much precision and robustness as possible, however, I will first consider an issue (in section 2) that may appear to be quite unrelated to underdetermination, namely the truth (or otherwise) behind the slogan "absence of evidence is not evidence of absence". As we shall see, a probabilistic analysis of the conditions under which the slogan comes out as true will help in determining what sorts of factors are relevant to how likely a theory is to be underdetermined (in the relevant sense).

\section{Evidence And Absence}

A popular slogan among scientists and statisticians has it that "absence of evidence is not evidence of absence". The slogan admits of many interpreta- 
tions. On one interpretation, the slogan holds that the fact that one hasn't come across something cannot even constitute part of one's evidence for the claim that no such thing exists. Thus interpreted, the slogan appears false: Consider your belief that there are no (genuine) pink elephants. How is this belief justified? It looks like an important piece of information justifying this belief may very well be that you have never seen a (genuine) pink elephant. After all, if you had seen a pink elephant, then you'd presumably not be justified in believing that there aren't any.

As Sober (2009) points out, this intuitive judgment is borne out in a Bayesian account of evidential support. On this account, $E$ confirms $H$ just in case $p(H \mid E)>p(H)$. The right-hand side of this biconditional is equivalent to $p(E \mid H)>p(E \mid \neg H)$, so on the Bayesian account anything one learns that is more likely to be the case given the truth of some hypothesis than given its negation confirms that hypothesis. Since one can surely be more likely not to have seen a pink elephant if there are no such things than if there are, it follows that on the Bayesian account the fact that one hasn't seen a pink elephant can confirm that there are none. Thus it looks like "absence of evidence" can be "evidence of absence" on the Bayesian account of confirmation. ${ }^{6}$

On a more charitable interpretation of "absence of evidence is not evidence of absence", the slogan holds that not having evidence that something exists is not sufficient for being rationally confident that it doesn't exist. Here the statement " $E$ is evidence of $H$ " is interpreted not as claiming that $E$ makes $H$ more likely than it would otherwise be, but as claiming that $E$ makes $H$ likely to be true - sufficiently likely, perhaps, for one to believe, accept, or be reasonably confident that $H$ is true. ${ }^{7}$ This point is illustrated by the pink elephant case, since the mere fact that one hasn't come across

\footnotetext{
${ }^{6}$ Sober (2009) also points out that there is something to the slogan that "absence of evidence is not evidence of absence" in that absence of evidence is usually not very strong evidence for absence, given a "likelihoodist" measure of strength of confirmation. As Strevens (2009) points out, however, this is hardly a completely satisfactory interpretation of the original slogan, for it contradicts the slogan's apparent meaning. I offer a different way of interpreting the slogan below - one that does not contradict the apparent meaning of the slogan.

${ }^{7}$ Salmon (1975) famously distinguished between these two interpretations, referring to the first as the "relevance concept of confirmation" and the second as the "absolute concept of confirmation".
} 
pink elephants is presumably not by itself sufficient to justify a reasonably high degree of confidence that there are no pink elephants. More generally, there are obviously many kinds of things that you shouldn't be confident don't exist even though you have never come across such things. So it looks like there is something to the idea that absence of evidence is not evidence of absence, if interpreted as the claim that the fact that one hasn't come across something is not sufficient for justifying a reasonably high degree of confidence that it doesn't exist.

Now, if the second version of the slogan is correct, but not the first, then absence of evidence may be part of of one's justification for being reasonably confident that things of that kind (probably) don't exist, but it cannot be the only part. This raises the following question: Provided that one knows that one has not come across something, when should one be reasonably confident that things of this kind don't exist? What other factors need to be present in order for "absence of evidence" to form part of one's justification for a reasonably high degree of confidence that the thing in question really is absent? A natural strategy for answering this question in a precise way, which follows Sober's cue, is to look to epistemological applications of probability theory. In the discussion that follows on realism and underdetermination, we will be giving these probabilities a frequency interpretation. For now, however, we can for convenience follow Sober and Bayesians more generally - in taking them to refer to rational degrees of confidence.

Let $F_{X}$ be the proposition that instances of some particular kind $X$ have been found or discovered, and let $A_{X}$ be the hypothesis that no things of kind $X$ exist. Given this, we can represent the rational degree of confidence that there are no $X \mathrm{~s}$ given the fact that no $X \mathrm{~s}$ have been found (and also given some background evidence $B$ ) as the conditional probability $p\left(A_{X} \mid \neg F_{X} \& B\right)$. The significance of this is illustrated by Bayes's Theorem:

$$
\begin{aligned}
& p\left(A_{X} \mid \neg F_{X} \& B\right)= \\
& \frac{p\left(\neg F_{X} \mid A_{X} \& B\right) p\left(A_{X} \mid B\right)}{p\left(\neg F_{X} \mid A_{X} \& B\right) p\left(A_{X} \mid B\right)+p\left(\neg F_{X} \mid \neg A_{X} \& B\right) p\left(\neg A_{X} \mid B\right)}
\end{aligned}
$$

Before we go further, let us simplify (1) in two ways. First, since the probabilities of a proposition and its negation always sum to one, we can replace $p\left(\neg A_{X} \mid B\right)$ with $\left(1-p\left(A_{X} \mid B\right)\right)$, and $p\left(\neg F_{X} \mid \neg A_{X} \& B\right)$ with 
$\left(1-p\left(F_{X} \mid \neg A_{X} \& B\right)\right)$. Second, consider $p\left(\neg F_{X} \mid A_{X} \& B\right)$ : This is the probability that one would not have found something of a particular kind given that there are no such things (and given background evidence $B$ ). Since "finding" is factive in the sense that one cannot find something of a kind that doesn't exist, $A_{X}$ entails $\neg F_{X}$. Hence the probability calculus dictates that $p\left(\neg F_{X} \mid A_{X} \& B\right)=1 .{ }^{8}$ Using some simple algebra, we can thus reformulate (1) as follows:

$$
p\left(A_{X} \mid \neg F_{X} \& B\right)=\frac{1}{1+\left(1-p\left(F_{X} \mid \neg A_{X} \& B\right)\right)\left(\frac{1}{p\left(A_{X} \mid B\right)}-1\right)}
$$

From (2) we see that the probability of $A_{X}$ if $\neg F_{X}$ is part of your evidence (and $B$ is your background knowledge) depends on two (and only two) factors:

(a) $p\left(A_{X} \mid B\right)$ : the probability of $A_{X}$ given only $B$,

(b) $p\left(F_{X} \mid \neg A_{X} \& B\right)$ : the probability of $F_{X}$ given $B$ and that $A_{X}$ is false.

More precisely, the probability of $A_{X}$ given $\neg F_{X}$ (and $B$ ) is higher to the extent that (a) and (b) are higher. This will help us see when not having found something makes it likely that something doesn't exist, so let's discuss these two factors in turn.

Regarding (a): This is the probability that there are no things of a certain kind independently of whether such things have been found. It should come as no surprise that the probability of there not being anything of a certain kind is positively dependent on this probability. Returning to our previous example, the probability of there not being any pink elephants given the fact that you've never come across any such things is clearly proportional to the probability of there not being any pink elephants regardless

\footnotetext{
${ }^{8}$ This simplification obviously depends on $F_{X}$ being the proposition that one has in fact found an $\mathrm{X}$ (e.g. as opposed to the proposition that one merely believes oneself to have found an $\mathrm{X}$ ). It is of course possible for an agent to be mistaken about whether she has found something of a given kind, e.g. as Rene Blondlot mistakenly believed in 1903 that he had discovered a new form of radiation, "N-rays". However, even an agent who believes herself to have found something that does not in fact exist should assign probability 1 to $p\left(\neg F_{X} \mid A_{X} \& B\right)$, since by her lights the conditional "If Xs did not exist, I would not have found any Xs" is still necessarily true. Such an agent, if rational, would not object to the conditional itself, but instead deny the consequent of the conditional and thus also its antecendent.
} 
of whether you have found any. Had you thought, for example, that there are a lot of pink mammals, or that elephants come in all sorts of colors, then you should be less confident that there are no pink elephants, quite independently of the fact that you haven't come across pink elephants.

Regarding (b): This is the probability that we would have found something of a particular kind given that such things exist. Again, it should come as no surprise that this is relevant to the probability of there being things of a given kind, for when we argue that something doesn't exist on the basis of us not having found any such thing, then we seem to be assuming that it's at least somewhat likely that we would have found such a thing if it existed. In our pink elephant case, it surely matters to the probability of pink elephants existing how probable one finds it that one would have come across pink elephants if they existed. This might depend on one's background beliefs, for example one whether one believes that there has been a substantial (but unsuccessful) search for pink elephants, diligently conducted by the Official Scout of the Pink Elephant Society.

Note that (2) illustrates that the probability that something doesn't exist might be high even when (a) is very low, provided that (b) is high enough to compensate: If $p\left(A_{X} \mid B\right)$ is very low, then $\left(\frac{1}{p\left(A_{X} \mid B\right)}-1\right)$ will be very high, but if $p\left(F_{X} \mid \neg A_{X} \& B\right)$ is high enough, then $\left(1-p\left(F_{X} \mid \neg A_{X} \& B\right)\right)\left(\frac{1}{P\left(A_{X} \mid B\right)}-1\right)$ can still be quite low, and so $p\left(A_{X} \mid \neg F_{X} \& B\right)$ would still be fairly close to 1 . The converse is true as well, of course: The probability that that something doesn't exist might be high even when (b) is very low, provided that (a) is high enough to compensate. Generally, on a probabilistic analysis, (a) and (b) are both relevant to how confident one should be that something doesn't exist, but a strong presence of one factor can compensate for a lack of the other. This is important because it reminds us of just how misleading it can be to focus on only one of the two factors, and thus mistakenly assume that any argument is weak if that factor is not strongly present. We need to look at the two factors in concert.

Now, how is all of this relevant to epistemic realism and RTU? To a first approximation - to be spelled out in the following section - the argument goes as follows: Consider a scientific theory $T$ for which we haven't come across any underdetermination rival. Since a theory is underdetermined (in the relevant sense) just in case it has such an underdetermination rival, the conditions under which a theory is likely not to have any underdeter- 
mination rival are precisely the conditions under which we should conclude the theory is unlikely to be underdetermined. Hence we can distinguish - in a principled and epistemically motivated way - between the theories that are more and less likely to be underdetermined based on the extent to which the probabilities here labelled (a) and (b) are present with regard to underdetermination rivals for such theories. This basic idea will constitute an important part of the my response to Stanford's New Induction.

\section{DETERMINING UNDERDETERMINATION}

In the previous section, I argued that as a general matter, the fact that we haven't found something of a given kind makes it likely that there are no such things depends on the combined extent to which (a) it is likely that something exists regardless of whether it has been found, and (b) it is likely that we would have found such things if they existed. We now apply this probabilistic lesson to the question of how likely it is that a given scientific theory has underdetermination rivals, i.e. (undiscovered) rival theories that are equally well supported given one's current evidence. Our aim will be to distinguish between theories that are more and less likely to be have such underdetermination rivals, for this (as I'll argue in the next section) will equip us with the tools to undermine the New Induction in a principled and non-question-begging manner.

In the previous section we were interested in a Bayesian analysis of the slogan "absence of evidence is not evidence of absence". Our interest now is not with absences of evidence, but with absences of underdetermination rivals. In particular, we are trying to locate a set of theories for which the fact that no underdetermination rivals have been found indicates that there are no such underdetermination rivals to be found - that the currently accepted theory is not just better supported by serious rivals theories we are aware of but also better supported than any rivals that we could come to be aware of. Another important difference between the preceding discussion and what follows is that we will now switch from a Bayesian interpretation of the probabilities to a frequency interpretation. So the probabilities to be discussed in what follows are the relative frequencies in the members of 
various sets of scientific theories. ${ }^{9}$ For example, the probability of something being in a set $\mathcal{A}$ given that it is in another set $\mathcal{B}, p(\mathcal{A} \mid \mathcal{B}),{ }^{10}$ signifies the frequency with which members of $\mathcal{B}$ are also in $\mathcal{A}$.

Now, let $\mathcal{A}_{U}$ be the set of hypotheses for which there are no underdetermination rivals, and let $\mathcal{F}_{U}$ be the set of hypotheses for which underdetermination rivals have been found. What we are interested in is finding a set $\mathcal{X}$ such that most theories in $\mathcal{X}$ for which underdetermination rivals have not been found do indeed not have any such rivals. Given a frequency interpretation of the following probabilities, we are in other words seeking an $\mathcal{X}$ such that $p\left(\mathcal{A}_{U} \mid \overline{\mathcal{F}_{U}}, \mathcal{X}\right)$ is high (where $\overline{\mathcal{F}_{U}}$ is the set of theories for which underdetermination rivals have not been found). In an exactly analogous way as in section 2 we have that, since no underdetermination rivals could possibly be found to theories for which there are no such underdetermination rivals at all, $p\left(\overline{\mathcal{F}_{U}} \mid \mathcal{A}_{X}, \mathcal{X}\right)=1 .{ }^{11}$ As before, then, we calculate $p\left(\mathcal{A}_{U} \mid \overline{\mathcal{F}_{U}}, \mathcal{X}\right)$ as follows:

$$
p\left(\mathcal{A}_{U} \mid \overline{\mathcal{F}_{U}}, \mathcal{X}\right)=\frac{1}{1+\left(1-p\left(\mathcal{F}_{U} \mid \overline{\mathcal{A}_{U}}, \mathcal{X}\right)\right)\left(\frac{1}{p\left(\mathcal{A}_{U} \mid \mathcal{X}\right)}-1\right)}
$$

Again this reveals that the probability in question depends on two and only two factors, which I will henceforth refer to as follows:

PLAUSIBILITY: $p\left(\mathcal{A}_{U} \mid \mathcal{X}\right)$; the frequency of theories for which there are no underdetermination rivals among the theories in $\mathcal{X}$ as a whole.

\footnotetext{
${ }^{9}$ This frequency interpretation is appropriate in the context of of evaluating arguments for RTU, which holds that most empirically successful theories are underdetermined - i.e., that the frequency of underdetermined theories is high among empirically successful theories.

${ }^{10}$ Here I am abusing notation by writing $p(\mathcal{A} \mid \mathcal{B})$ instead of $p(x \in \mathcal{A} \mid x \in \mathcal{B})$.

${ }^{11}$ Of course, this is not to say that one could not mistakenly believe oneself we have found an underdetermination rival to a given theory even when no such rivals exist. While the possibility of mistakenly believing oneself to have found an underdetermination rival does not speak against the fact that $p\left(\overline{\mathcal{F}_{U}} \mid \mathcal{A}_{U}, \mathcal{X}\right)=1$ - which is a necessary truth since it is impossible to find something of a kind that does not exist - it does highlight the fact that it is assumed in the debate as a whole that it is possible to reliably estimate whether a given theory that has already been discovered is indeed an underdetermination rival to another theory. Indeed, this assumption is essential to the New Induction's argumentative strategy since if we could not reliably locate historical cases of underdetermination rivals then proponents of the New Induction could not argue that they are sufficiently common in the history of science to inductively warrant the conclusion that most current successful theories are underdetermined as well.
} 
SENSITIVITY: $p\left(\mathcal{F}_{U} \mid \overline{\mathcal{A}_{U}}, \mathcal{X}\right)$; the frequency of theories for which an underdetermination rival has been discovered among theories in $\mathcal{X}$ for which such rivals exist. ${ }^{12}$

Put in terms of probabilities, we can say that the probability that a given theory in $\mathcal{X}$ has underdetermination rivals depends on (i) the probability that there are such rivals to theories in $\mathcal{X}$ regardless of whether they have been found (PLAUSiBILITY), and (ii) the probability that we would have found such rivals to theories in $\mathcal{X}$ if they existed (SEnsitiviTy). Thus let us examine each of the two factors in turn to see what it reveals about how to construct a set $\mathcal{X}$ for which theories are least likely to be underdetermined.

PLAUSIBILITY is a measure of how frequently a theory in $\mathcal{X}$ as a whole has no underdetermination rivals. Now, note that to the extent that a given theory is well supported by the available evidence - i.e. empirically successful - it is also unlikely that a rival theory is better supported than it is. Thus, all other things being equal, the empirical success enjoyed by a given theory increases the PLAUSIBILITY that no other theory is an underdetermination rival to it. For this reason, realists may insist that the very fact that empirically successful theories are successful makes them unlikely to have underdetermination rivals. This might be taken to support the realist thesis that empirically successful theories are unlikely to be underdetermined. In particular, then, since currently accepted theories are arguably much more successful than their now-discarded counterparts, realists may argue that current theories are much less likely to be underdetermined. ${ }^{13}$

Realists may be right about this point, but it will hardly convince those who are sympathetic with anti-realism to begin with. After all, the claim

\footnotetext{
${ }^{12}$ I have deliberately chosen terms for these two factors that are not widely used in the literature on scientific theory testing in order to prevent them from being confused with a number of factors discussed in that literature. For example, although SENSITIVITY is superficially similar to "statistical power", i.e. the probability that a false null hypothesis will be rejected in an empirical test, it would be misleading to refer to $p\left(\mathcal{F}_{U} \mid \overline{\mathcal{A}}_{U}, \mathcal{X}\right)$ as a given theory's "statistical power" since that falsely suggests that the theory has this kind of statistical power with regard to a set of results from an empirical test. After all, note that SENSITIVITY is concerned with the probability that a group of scientists discover underdetermination rivals to theories in a given set, which is a feature that does not depend directly on the features of any empirical test.

${ }^{13}$ This argument is implicit in many realist responses to the New Induction, but Devitt (2011) arguably provides the clearest and most careful objection of this sort.
} 
that empirically successful theories are unlikely to be underdetermined is precisely the point that anti-realists have been in the business of denying ever since Laudan's (1981) historical challenge to realism. Anti-realists of this stripe will argue that empirical success is a poor guide to PLAUSIBILITY by appealing to the many cases from the history of science of very successful theories that later turned out to have underdetermination rivals. Indeed, this is exactly what Stanford (2006) aims to do with his New Induction. We will examine this argument in more detail below, but my point for now is that to assume that more empirically successful theories have a higher degree of PLAUSIBILITY is at best to beg the question against the antirealist. The response outlined in this paper, by contrast, has the advantage of not assuming that PLAUSIBILITY is higher for more successful theories than for their less successful counterparts. Thus the response will provide a non-question-begging way of avoiding the New Induction.

To see how this response works, note that our analysis of when "absence of evidence" is "evidence of absence" shows that a theory may be unlikely to be underdetermined even if PLAUSIBILITY is very low - provided that SENSITIVITY is high enough to compensate. For example, suppose for example that $p\left(\mathcal{A}_{U} \mid \mathcal{X}\right)=0.2$ and $p\left(\mathcal{F}_{U} \mid \overline{\mathcal{A}_{U}}, \mathcal{X}\right)=0.9$. Now, by plugging these numbers into equation $(3)$ we get $p\left(\mathcal{A}_{U} \mid \overline{\mathcal{F}_{U}}, \mathcal{X}\right) \approx 0.714$. This illustrates that the fact that a given set has a low PLAUSIBILITY does not by itself show that the theories in the set are likely to be underdetermined, since the set's SENSITIVITY may compensate for this. So we have in SENSITIVITY a principled rationale for distinguishing between sets of theories that are more and less likely to be underdetermined that does not beg the question against the epistemic anti-realist.

Now, recall that SENSITIVITY is here the frequency among underdetermined theories in $\mathcal{X}$ of theories for which an underdetermination rival has been discovered. Put in terms of probabilities, SENSITIVITY is the probability of that we would have found underdetermination rivals to a given theory in $\mathcal{X}$ given that such a rival exists. But what kind of factors indicate whether that is the case for a given set of theories $\mathcal{X}$ ? Clearly, the kinds of factors that are relevant will depend to a large extent on those who are looking for the theories - i.e. on the scientists themselves and the communities they form. For that reason, we shouldn't expect to find any kind of algorithm determining SEnsitiviTy. At best, we can try to iden- 
tify some such factors given assumptions about the nature and structure of scientific communities - assumptions that may very well be called into question. That said, it seems to me that it ought to be uncontroversial that the following four factors will, ceteris paribus, indicate that a set of theories has a higher or lower degree of SENSITIVITY:

(i) Domain: First of all, the nature of the theoretic domain itself will be relevant to how difficult it is to discover underdetermination rivals if there are any. As emphasized by both (Roush, 2005, 211-12) and (Stanford, 2009), some areas are such that, due to various psychological barriers, it is more difficult to construct theories in those areas. For example, if a domain of inquiry calls for very complicated theories, then it may be harder to conceptualize what alternatives to theories in that domain would look like. Additionally, some domain may call for theories that are simply quite alien to our everyday ways of conceptualizing the world, in which case it may be harder to conceive of what plausible theories in that domain would be like. If it is true, for example, that our way of conceptualizing the world is basically Newtonian, then domains that call for radically non-Newtonian thinking (e.g. cosmology and subatomic particle physics) may be such that underdetermination rivals are more likely to remain undiscovered. ${ }^{14}$

(ii) Anti-dogmatism. Second, the extent to which scientists are willing to consider alternative theoretical approaches in their fields will of course be a factor in determining the probability of finding an underdetermination rival to a given theory, if there is one. Now, followers of Kuhn $(1996,1977)$, will no doubt urge that in so far as theories that are widely accepted and fundamental to a scientific discipline, scientists will have to rely heavily on them in their daily investigations, and so have less of an incentive to discover rivals to them. If so, then there would be little incentives to discover rivals to fundamental and widely accepted theories, leading to a kind of dogmatism with

\footnotetext{
${ }^{14}$ Thus, assuming that unobservables are, all other things being equal, harder to conceptualize, there is some truth to the anti-realist claim that theories that concern unobservables are, all other things being equal, more likely to be underdetermined. However, as I'll emphasize below, other things are not always, or even usually, equal, and so theories about unobservables can very well be less likely to be underdetermined than theories about observables.
} 
regard to accepted theories. ${ }^{15}$ However, it has also be argued that alternative theories often play an indispensable role in both developing and testing the theories that we already consider most promising (Feyerabend, 1963), and that the imperative to consider new theoretical approaches is an essential part of the scientific ethos (Merton, 1973). ${ }^{16}$ While my sympathies lie squarely with the second viewpoint, I shall not argue for it here or assume it in what follows. Instead, I shall grant for the sake of the argument that individual scientists may exhibit considerable dogmatism with regard to alternative theoretical approaches, and argue that Stanford's New Induction can still be undermined.

(iii) Community. Third, the nature and size of the scientific community working with a theory will clearly be relevant for how likely it is that there are no underdetermination rivals to a theory if it hasn't yet been found. Most obviously, the more scientists there are in a given field the more likely it is that one of them has found, or perhaps stumbled upon, an underdetermination rival to a given theory if it existed. Relatedly, some communities of scientists will be more skilled at theorizing than others, e.g. because they possess greater background evidence that influences their conception of possible theoretical frameworks. Scientific theorizing (like philosophical theorizing) is a skill that can be gained by training and selected for in various social selection processes (e.g. in applications for graduate school, professorships, and research funding).

(iv) Time. A fourth factor is simply the period of time a theory has been accepted or contemplated. A theory that has been considered for a long time, especially if widely accepted during that time, can be expected to have a richer history of searches for rivals and use in various scientific and ordinary contexts. Thus, the longer a theory has been accepted or considered by a scientific community, the likelier it is that the fact that we haven't found any underdetermination rivals

\footnotetext{
${ }^{15}$ See Magnus (2010) for an argument along these lines.

${ }^{16}$ Indeed, anti-dogmatism is really just compliance with the norm of "organized skepticism", which the eminent sociologist of science Robert Merton argued was one of the four norms comprising the scientific ethos.
} 
to it is due to the fact that there are none to be found.

We have identified four factors which plausibly indicate SENSITIVITY, and thus the probability that a given empirically successful theory for which no underdetermination rivals have been found will not have any such rivals: (i) domain, (ii) anti-dogmatism, (iii) community, and (iv) time. I call these "identifiable indicators" of SENSITIVITY in order to emphasize that, in contrast to SENSITIVITY itself, they are factors which can be fairly easily identified in historical case-studies. As such, they may provide a rough guide to SENSITIVITY in historical cases where we obviously do not have direct access to the probability that an underdetermination rival would have been discovered if it existed.

Of course, this ease of identification comes at a price, in that these factors are less precise and more intricate than the more precisely defined and rigorously motivated SENSITIVITY. However, it seems undeniable that each of these factors provides at least some indication, ceteris paribus, of a given theory's SEnsitiviTy. For example, it would be more than a little implausible to insist that a larger and more competent community of scientists is not, ceteris paribus, more likely to have discovered an underdetermination rival to a given theory if such a rival existed. Thus, while we may disagree on how strong an indication of SENSITIVITY each of these factors provide, it can hardly be contended that these factors are irrelevant to SENSITIVITY. Note also that the four factors will often be synergetic, combining to produce an effect on SENSITIVITY that may be greater (or lesser) than the sum of their parts. As indicated above, I will not attempt to provide any algorithm for agglomerating these factors into an overall estimation of SENSITIVITY. This is not only because I do not believe any such agglomeration procedure could reasonably be provided, but also because (as we shall see below) no such agglomeration is needed for the argument below to go through.

At any rate, it's important to note that, as with SENSITIVITY itself, all of the identifiable indicators come in degrees. As a consequence, the risk of underdetermination for a given theory will also be a matter of degree. Only in very exceptional cases can we be absolutely sure either that our theory is underdetermined, or that it's not. So the point of the identifiable indicators is not to find a way to guarantee that one's theories are not underdetermined, but to distinguish between theories that are more and less likely to 
be underdetermined. Indeed, in what follows I will not be assuming that identifiable indicators can tell us the particular degree of SENSITIVITY had by a given theory. Rather, I shall assume only that we can use the identifiable indicators as our guide in identifying some theories as having a higher degree of SENSITIVITY than other theories. In other words, I'll use the identifiable indicators only as guides to comparative rather than absolute degrees of SENSITIVITY. As it turns out, these comparative evaluations of SENSITIVITY will suffice in undermining Stanford's New Induction.

\section{The NeW Induction}

The New Induction begins with the undeniable historical observation that for a great many theories that were accepted in the past, often because they were very successful, later investigation would reveal alternative theories that were equally well supported given the available evidence. In short, these theories turned out to have underdetermination rivals (in the sense spelled out in section 1). From this it is inferred by a simple enumerative induction that the typical situation in science is that even empirically successful theories - including those that are currently accepted - have "unconceived" (i.e. undiscovered) underdetermination rivals. In other words, the argument concludes that empirically successful theories are typically underdetermined, which of course is what the relevant underdetermination thesis, RTU, claims. (Although versions of this argument have been advanced by Hesse (1976), Sklar (1981), Laudan (1981), and Roush (2005), I shall focus on the version given by Stanford (2006), since his is arguably the most developed, sophisticated and widely-discussed version.)

What makes the New Induction so challenging for realists is that it promises to undermine the notion that we are justified in believing even those theories that are truly empirically successful, on any plausible definition of "empirical success". The reason for this is that the inductive base of the New Induction consists of theories that, undeniably, enjoyed great empirical successes. For example, Fresnel's ether theory of light was hugely successful, even by realists' lights, and yet it was superseded by what is arguably a better supported theory a few decades later. (I will return to this example shortly.) The New Induction concludes that empirical success is 
a poor indicator of whether a theory is underdetermined, and thus that we are not justified in believing even very successful scientific theories to be true, at least not "in those parts of the sciences that seek to theorize about the fundamental constitutions of the various domains of the natural world and the dynamical principles at work in those domains". (Stanford, 2006, 32)

As I indicated towards the end of section 1, my strategy for responding to this argument will be to identify a proper subset of empirically successful theories for which the induction is weakest. It should be clear by now what that proper subset is: It is the set of successful theories for which SENSITIVITY is higher than the now discarded (but at the time successful) theories which turned out to have unconceived underdetermination rivals. Since a successful theory with higher SENSITIVITY is, as we have seen, less likely to be underdetermined than an otherwise similar theory with lower SENSITIVITY, an inductive argument from a set of theories with lower SENSITIVITY to those with higher SENSITIVITY will clearly be a weak argument. (Compare: Since I know that it is less likely to rain when the air pressure is higher, an argument from the fact that it rained the past three days to the conclusion that it will rain today is weak if my barometer tells me that the air pressure rose this morning.)

Of course, this point will only be interesting if there are at least some currently accepted theories for which SENSITIVITY is indeed higher than for the theories that form the inductive base of Stanford's New Induction. To see why there are such theories, it will be useful to consider the "identifiable indicators" of SENSITIVITY for underdetermination rivals that I proposed in the previous section. In order for Stanford's argument to support the claim that a given presently accepted successful theory $T$ is likely to be underdetermined, one would need to specify an inductive base containing theories for which domain, anti-dogmatism, community, and time indicate a greater or similar level of SENSITIVITY. I submit that we can be rather optimistic that for many of our currently accepted theories there will simply not be enough now-discarded theories to figure in the skeptic's inductive base. To illustrate the point, I will briefly consider two cases from the history of science that have been taken to be especially problematic for scientific realism.

Consider first Augustin-Jean Fresnel's ether theory of light, which was 
later replaced by James Clerk Maxwell's electromagnetic theory. ${ }^{17}$ The relevant question is: How reasonable was it to believe that we would have found underdetermination rivals to Fresnel's theory if they existed (as we now know that they did)? ${ }^{18}$ To focus the discussion, let us consider Fresnel's claim that light travels as a wave in a material medium. As is well documented, Francois Arago showed in 1818 (to Simeon Poisson's great surprise) that Fresnel's theory's prediction that a bright spot would appear at the center of the shadow of an opaque disc was indeed correct. In 1855, however, Maxwell presented a rival theory to that of Fresnel, in which it was posited that the material in which light waves travel was a complicated system of molecular vortices (later to be replaced by the electromagnetic field, yielding Maxwell's famous electromagnetic theory of light).

Now, consider Fresnel's claim just before Maxwell's presentation of a rival theory of the medium of light waves in 1855. My claim is that Fresnel's situation was dissimilar to the situation we are in today with regard to many currently accepted theories. Consider, for example, the currently accepted theory of light - the photon theory (as I will call it) - on which light consists of collections of photons with a "dual nature" of both electromagnetic waves and discrete particles. How does the photon theory compare with Fresnel's with regard to each of the identifiable indicators of SENSITIVITY?

Let's consider the four factors in turn: (i) First, the domain of inquiry of each theory is the same, of course, since they are theories about the very same things. (ii) Second, although it is hard to evaluate the antidogmatism exhibited by scientists in different time periods, there is no reason to think Fresnel's contemporaries were less prone to being dogmatic about their theories than the optical physicist of the 20th and 21st century. Indeed, a study conducted in 2002 suggests that around $90 \%$ of scientists believe that the scientists should "consider all new evidence, hypotheses, theories, and innovations, even those that challenge or contradict their own work." (Anderson et al., 2010, 7) For obvious reasons, there are no

\footnotetext{
${ }^{17}$ This is one of the most widely discussed cases of empirically successful theories for which it turned out that underdetermination rivals existed. See, for example, (Worrall, 1989, 1994), (Psillos, 1999), and (Chakravartty, 2007).

${ }^{18}$ Note that the question is not whether it's reasonable to believe now that Fresnel's theory had such rivals. The question is whether it was reasonable at the time.
} 
comparable studies from Fresnel's time, but this study suggest that current scientists are unlikely to be more dogmatic than Fresnel's contemporaries. ${ }^{19}$ (iii) Third, the community of theoretical physicists has grown and matured significantly since 1855. Indeed, based on the exponential growth of journal articles in physics (with a doubling time of around 10-15 years) and similar trends in the number of working scientists cited in biographical records of scientists, we have good reason to believe that the number of working physicists has increased exponentially as well. (Price, 1961, 1963; Larsen and von Ins, 2010) Moreover, given the increasing professionalization of physical science since the late 19th century (see, e.g., Bockstaele, 2004), contemporary physicists will certainly have a greater stock of background knowledge, better access to relevant research, and a more rigorous formal education than the scientists of the early 19th century. As a case in point, we may note that Fresnel himself was not trained specifically as an optical physicist, and started his career as a chemist before switching to optical physics a few years before presenting his ether theory. (Buchwald, 1989, 113-114) (iv) Fourth, the photon theory has undeniably been considered for a much longer time than Fresnel's theory had in 1855: The photon theory was first formulated by Einstein in 1905, and so has been considered for well over a century, whereas Fresnel's theory was first formulated in 1818 and so had only been considered for less than four decades in 1855. (Buchwald, 1989) So, in sum, domain and anti-dogmatism favors neither the photon theory nor the ether theory, while community and time both strongly favor the photon theory.

This is not an isolated case of a currently accepted theory being dissimilar with respect to SENSITIVITY to the theories that form the inductive base of the New Induction. In fact, the same point can be made about Stanford's central case study, viz. theories of biological heredity in the 19th century. (Stanford, 2006, chapters 3-5) At least three serious theories were proposed, each one with at least some degree of empirical success: Charles Darwin's "pangenesis" theory, which was replaced by Francis Galton's "stirp" theory,

\footnotetext{
${ }^{19}$ Of course, current scientists may be more confident in the photon theory than Fresnel's contemporaries were in his ether theory, but we shouldn't confuse confidence in theories with a dogmatic attitude towards alternative approaches. Besides, the photon theory itself underwent a significant period in the early 20th century where there was widespread doubt about the truth of the theory.
} 
which in turn was replaced by August Weismann's "germ-plasm" theory. Now, Stanford points out that Darwin, Galton and Weismann were all apparently unable to conceive of the theory that would later replace their own. But does this support the conclusion that our currently accepted theory of heredity - roughly, a version of Mendelian genetics - will also one day be replaced by a rival that is at least as well supported by the available evidence? Only if Darwin's, Galton's and Weismann's theories had, at the time of their acceptance, a degree of SENSITIVITY that is comparable to that which Mendelian genetics has today. ${ }^{20}$

So let's consider briefly how the theories compare with regard to the four identifiable indicators of SENSITIVITY: (i) First, all of these theories are in the same domain, so there is no difference in that regard between Mendelian genetics and the three alternative theories of heredity. (ii) Second, there is again no reason to think that scientists who accept Mendelian genetics are, as a rule, more dogmatic than those following Darwin, Galton and Weismann in the 19th century, so there is no plausible difference with regard to anti-dogmatism. (iii) Third, the scientific community of biologists working on heredity was of course significantly smaller than it is now, which is again evidenced by an exponential growth in published journal articles in biology and similar trends in the number of working scientists cited in biographical records. (Price, 1961, 1963; Larsen and von Ins, 2010) Furthermore, the study of genetics and heredity in particular was in its infancy as a profession when Darwin, Galton and Weismann were proposing their theories in the late 19th century. ${ }^{21}$ This fact is noted by Ernst Mayr in his discussion of the progression of biology from the 19th century onwards in The Growth of Biological Thought:

In many disciplines and subdisciplines, there was only a single specialist at any one time. So few people were working in the different branches of biology that Darwin thought he could afford to wait twenty years before publishing his theory of natural

\footnotetext{
${ }^{20} \mathrm{I}$ am assuming here that Mendelian genetics is at least as successful empirically as each of the other theories, and thus that it can be assumed to have at least as high PLAUSIBILITY.

${ }^{21}$ As a case in point, the genetics societies of Britain and the United States were not founded until 1919 and 1931 respectively. The official journals of each society, Heredity and Genetics, were established in 1947 and 1916 respectively.
} 
selection. He was quite thunderstruck when somebody else (A. R. Wallace) had the same idea. When the professionalization of biology began with the establishment of chairs for diverse branches of biology at many universities and when each professor began educating numerous young specialists, an exponential acceleration in the rate of scientific production occurred. (Mayr, $1982,111)$

(iii) Fourth, there is a significant difference with regard to time: Mendelian genetics has been accepted, in one form or another, for at least a century, whereas all of the theories Stanford discusses - Darwin's, Galton's and Weismann's - were presented to, and rejected by, the scientific community within the space of around three decades (roughly from 1868, when Darwin first presented his pangenesis theory, to the turn of the century, when Mendelian genetics first became widely known). (Bowler, 1989) So, in sum, we again have that domain and anti-dogmatism favors neither Mendelian genetics nor any of the other three theories, while community and time both strongly favor Mendelian genetics.

Of course, much more could be said about these cases to illustrate more fully how the four identifiable indicators are realized in concrete terms. My point, however, is a general one - illustrated but not constituted by these two cases - viz., that since SENSITIVITY is indicated by historical and sociological factors such as the amount of time a theory has been accepted and the size and structure of the community that accepts it, many of the theories that we accept today quite clearly have a higher SENSITIVITY than those theories that are used by Stanford and other anti-realists to infer that scientific theories are typically underdetermined. This is a distinction that really makes a difference, since theories with higher SENSITIVITY are less likely to be underdetermined, all else being equal. Note that this is not to deny that one can find some empirically successful and widely accepted theories about which the historical record suggests that we should be skeptical, e.g. theories that have recently been proposed, form part of some "fringe-science", or that concern some aspects of reality that we have reason to believe is exceptionally hard to conceptualize. Rather, the point is that there will be a suitably restricted subset of empirically successful and currently accepted theories for which the New Induction loses its original force. 
I'll now make a few clarificatory remarks in order to circumvent some objections. First, it must of course be conceded that there are historical cases in which an empirically successful theory with an apparently high degree of SENSITIVITY (as measured by the identifiable indicators) did turn out to have an underdetermination rival. The obvious example here is Newtonian mechanics, which was replaced by Einstein's theories of special and general relativity after being accepted for a long time by a fairly large scientific community. One might take such cases to illustrate that SENSITIVITY may be low even when the identifiable indicators are present to a high degree. But of course, we should expect some theories with high SENSITIVITY to be underdetermined, since a high degree of SENSITIVITY is not meant to guarantee that a theory is not underdetermined. So as long as cases like Newtonian Mechanics are rare among those that satisfy the four indicators of SENSITIVITY, as they do indeed seem to be, the mere existence of some such cases poses no threat to the claim that the four identifiable indicators provide a reliable guide to theories with high SENSITIVITY. Indeed, the very fact that such cases are rare in the history of science might be taken to support the conclusion that these indicators jointly capture the most important respects in which SENSITIVITY presents itself for scientific theories. $^{22}$

Second, note that the strength of this response to Stanford's New Induction, like Stanford's induction itself, will be a matter of degree. My strategy in this paper has been to undermine Stanford's induction by showing that the inductive base - consisting of various empirically successful theories accepted in the past - is dissimilar from the inductive target - consisting of various empirically successful and currently accepted theories - in a respect that bears crucially on the probability that the inductive target will have the relevant property if the inductive base does - where the relevant property is that of being probably underdetermined. Now, how much does this undermine the New Induction? Well, that clearly depends on how dissimilar the inductive target is to the inductive base in the relevant respect,

\footnotetext{
${ }^{22}$ Compare: Suppose I claim that most Swedish residents are protestants. It is no good objection to my claim to point out that Petersen, a Swedish resident, is catholic - especially not if the overwhelming majority of other Swedes that we have met have been protestants. That some of the Swedes we run into are non-protestants is exactly what we should expect if I were right that most (but not all) of them are protestants.
} 
i.e. on how much more SENSITIVITY can be claimed for present theories as compared with their past counterparts.

Assuming that we agree on the historical facts, this issue will turn on how well the identifiable indicators listed in the previous section track SENSITIVITY. Opinions will no doubt be divided on this issue. Some will agree with the present author that the greater time and larger, more qualified community of the photon theory as compared with the ether theory of light, for example, indicates a significant difference in SENSITIVITY and thus that an inductive argument from one to the other is very weak indeed. But even those who disagree about this point must surely acknowledge that many currently accepted theories have, in light of factors such as greater time and larger community, at least somewhat higher SENSITIVITY than their past counterparts. Now, while a greater difference in SENSITIVITY undermines the New Induction to a greater extent, any difference in SENSITIVITY undermines it to some extent, so even the most skeptical reader must acknowledge that the New Induction has been undermined (albeit perhaps only moderately) for those current theories.

\section{Conclusion}

As I have emphasized throughout, the key notion in this paper is SENSITIVITY - the extent to which it's plausible that one would have found things of a certain kind given that there are such things. When applied to what I called "underdetermination rivals", i.e. theories that are equally well supported by one's current evidence than accepted theories, I argued that SENSITIVITY varies with four "identifiable indicators", viz. the psychological accessibility of the domain of the theory in question, the anti-dogmatism with regard to alternative theoretical approaches, the nature of the community that potentially discovers such theories, and finally the time that a theory has been accepted or considered. My response to the New Induction, in brief, was that even if it is assumed (with the anti-realist) that the high level of empirical success of our current theories is an unreliable indicator of the existence of undiscovered underdetermination rivals, the in-

ductive inference is weak for a suitably restricted set of currently accepted theories because we have good reasons to think that that SENSITIVITY is 
(considerably) higher for those theories than for their counterparts in the New Induction's inductive base.

Note that to a large extent the "identifiable indicators" I discussed concern the history and sociological structure of scientific communities. Such factors have tended to downplayed by scientific realists, and emphasized by what may be described as their polar opposites - so-called "social constructivists" about science. One of the upshots of this paper is that realists may well have done themselves a disservice in this respect. Instead of avoiding the history and sociology of scientific communities, realists should see investigations in these fields as potential sources of considerations in defense of scientific realism. In this paper, I have identified the kind of factors that will be relevant in this regard, and shown how at least two currently accepted scientific theories (the photon theory of light and Mendelian genetics) compare (very) favorably in this regard to the past theories that the New Induction takes as its inductive base.

Relatedly, I have suggested that a plausible version of epistemic scientific realism - one that avoids Stanford's New Induction - is a "selective" realism that selects as justified those claims made by successful scientific theories that enjoy a certain kind of privileged status within scientific communities. Given the importance of the nature of scientific communities in this regard, this paper can be seen as taking the first steps towards a distinctively social scientific realism. That said, readers will note that I have not here given a positive argument for this realist position of the kind that many epistemic realists take themselves to possess in the form of the well-known No-Miracles Argument. Although a detailed discussion of whether such an argument can be given lies outside the scope of this paper, I will note that I am myself skeptical of "wholesale" arguments for realism of this sort, and instead favor "piecemeal" arguments for specific theories being justified based on the evidence that scientists themselves appeal to in a given case. ${ }^{23}$ Thus, in my view, the most important philosophical work for any epistemic realist consists in responding to the anti-realist arguments that are meant to undermine the support for scientific theories provided by the scientific evidence itself. It is this sort of work that I have undertaken here.

\footnotetext{
${ }^{23}$ Here I am in agreement with, among others, Blackburn (2002) and Magnus and Callender (2004).
} 


\section{REFERENCES}

Anderson, M. S., Ronning, E. A., DeVries, R., and Martinson, B. C. (2010). Extending the mertonian norms: Scientists' subscription to norms of research. Journal of Higher Education, 81:366-393.

Blackburn, S. (2002). Realism: Deconstructing the Debate. Ratio (new series), 15:111-133.

Bockstaele, P. (2004). The mathematical and the exact sciences. In Ruegg, W., editor, A History of the University in Europe Vol. III: Universities in the Nineteenth and Early Twentieth Centuries (1800-1945), pages 493518. Cambridge University Press, Cambridge.

Bowler, P. J. (1989). The Mendelian Revolution: The Emergence of Hereditarian Concepts in Science and Society. John Hopkins University Press, Baltimore.

Buchwald, J. Z. (1989). The Rise of the Wave Theory of Light: Optical Theory and Experiment in the Eearly Nineteenth Century. University of Chicago Press, Chicago, IL.

Cartwright, N. (1983). How the Laws of Physics Lie. Oxford University Press, Oxford.

Chakravartty, A. (2007). A Metaphysics for Scientific Realism: Knowing the Unobservable. Cambridge University Press, Cambridge.

Devitt, M. (2011). Are Unconceived Alternatives a Problem for Scientific Realism. Journal for General Philosophy of Science, 42:285-293.

Feyerabend, P. (1963). How to Be a Good Empiricist: A Plea of Tolerance in Matters Epistemological. In Baumrin, B., editor, Philosophy of Science: The Delaware Seminar. Interscience Publishers, New York, NY.

Gillies, D. (1993). Philosophy of Science in the Twentieth Century. Blackwell Publishers, Oxford.

Glymour, C. (1977). The Epistemology of Geometry. Noûs, 11:227-251.

Hacking, I. (1983). Representing and Intervening: Introductory Topics in the Philosophy of Natural Science. Cambridge University Press, Cambridge. 
Hesse, M. (1976). Truth and the Growth of Scientific Knowledge. In PSA: Proceedings of the Biennial Meeting of the Philosophy of Science Association, pages 261-280.

Kitcher, P. (1993). The Advancement of Science: Science without Legend, Objectivity without Illusions. Oxford University Press, New York.

Kitcher, P. (2001). Real Realism: The Galilean Strategy. The Philosophical Review, 110:151-197.

Kuhn, T. S. (1962/1996). The Structure of Scientific Revolutions. University of Chicago Press, Chicago, IL, 3rd edition.

Kuhn, T. S. (1977). Objectivity, Value Judgments, and Theory Choice. In The Essential Tension. University of Chicago Press, Chicago, IL.

Kukla, A. (1993). Laudan, Leplin, Empirical Equivalence and Underdetermination. Analysis, 53:1-7.

Larsen, P. O. and von Ins, M. (2010). The rate of growth in scientific publication and the decline in coverage provided by science citation index. Scientometrics, 84:575-603.

Laudan, L. (1981). A Confutation of Convergent Realism. Philosophy of Science, 48:19-49.

Laudan, L. (1990). Demystifying Underdetermination. In Savage, C. W., editor, Scientific Theories, Minnesota Studies in the Philosophy of Science, vol. 14. University of Minnesota Press, Minneapolis, MT.

Leplin, J. and Laudan, L. (1993). Underdetermination Underdeterred: Reply to Kukla. Analysis, 53:8-16.

Magnus, P. (2010). Inductions, Red Herrings, and the Best Explanation for the Mixed Records of Science. British Journal for the Philosophy of Science, 61:803-819.

Magnus, P. and Callender, C. (2004). Realist Ennui and the Base Rate Fallacy. Philosophy of Science, 71:320-338.

Mayr, E. (1982). The Growth of Biological Thought: Diversity, Evolution, and Inheritance. Harvard University Press, Cambridge, MA.

Merton, R. K. (1973). The normative structure of science. In Storer, N. W., editor, The Sociology of Science, pages 267-278. University of Chicago 
Press, Chicago, IL.

Poincaré, H. (1952). Science and Hypothesis. Dover, New York. Republication of the first English translation, published by Walter Scott Publishing, London, 1905.

Price, D. J. d. S. (1961). Science since Babylon. Yale University Press, New Haven.

Price, D. J. d. S. (1963). Little Science, Big Science. Columbia University Press, New York.

Psillos, S. (1999). Scientific Realism: How Science Tracks Truth. Routledge, London.

Putnam, H. (1978). Meaning and the Moral Sciences. Routledge and Kegan Paul, Boston.

Roush, S. (2005). Tracking Truth: Knowledge, Evidence, and Science. Clarendon Press, Oxford.

Salmon, W. C. (1975). Confirmation and relevance. In Maxwell, G. and Anderson, R., editors, Minnesota Studies in the Philosophy of Science, Vol. 6, Induction, Probability, and Confirmation, pages 3-36. University of Minnesota, Minneapolis, MT.

Sklar, L. (1981). Do Unborn Hypotheses Have Rights? Pacific Philosophical Quarterly, 62:17-29.

Sober, E. (2009). Absence of Evidence and Evidence of Absence: Evidential Transitivity in Connection with Fossils, Fishing, Fine-tuning, and Firing Squads. Philosophical Studies, 143:63-90.

Stanford, P. K. (2001). Refusing the Devil's Bargain: What Kind of Underdetermination Should We Take Seriously? Philosophy of Science (Proceedings Supplement), 68:S1-S12.

Stanford, P. K. (2006). Exceeding Our Grasp: Science, History, and the Problem of Unconceived Alternatives. Oxford University Press, Oxford.

Stanford, P. K. (2009). Scientific Realism, the Atomic Theory, and the Catch-All Hypothesis: Can We Test Fundamental Theories Against All Serious Alternatives. British Journal for the Philosophy of Science, 60:253-269. 
Stanford, P. K. (2013). Underdetermination of Scientific Theory. In Zalta, E. N., editor, Stanford Encyclopedia of Philosophy (Winter 2013 Edition).

Strevens, M. (2009). Objective Evidence and Absence: Comment on Sober. Philosophical Studies, 143:91-100.

Worrall, J. (1989). Structural Realism: The Best of Both Worlds. Dialectica, 43:99-124.

Worrall, J. (1994). How to Remain (Reasonably) Optimistic: Scientific Realism and the "Luminiferous Ether". In Richard M. Burian, D. H. and Forbes, M., editors, PSA 1994: Proceedings of the 1994 Biennial Meeting of the Philosophy of Science Association, volume 1, pages 334-342.

Wray, K. B. (2011). Epistemic Privilege and the Success of Science. Nous, $46: 375-385$. 